\title{
A Model of Immune Suppression and Repertoire Evolution
}

\author{
John Burns and Heather J. Ruskin \\ Dublin City University, Dublin 9, \\ Ireland \\ \{jburns, hruskin\}@computing.dcu.ie \\ http://www.dcu.ie/computing/msc/index.html
}

\begin{abstract}
We present a stochastic cellular automata model that allows us to study both localized and generalized aspects of the immune system (IS). We show how critical values for T Cell Receptor (TCR) affinity and cross-reactivity $(\rho)$ can determine the course of a viral infection. The model presented here offers insight into the widely varying pathology of infectious agents across individuals. Additionally, our model points to ways in which auto-immune disease can occur. We show that by integrating models of physical space and shape space we can analyze immune repertoire evolution and distribution over various time periods ranging from a few days up to three years.
\end{abstract}

\section{Introduction}

One of the questions that has long exercised immunological researchers is why different people (or, more precisely, their immune systems) respond differently to the same viral or antigen challenge. This question is exemplified by the pathology of the Human Immune Virus (HIV). In particular, the progression of the HIV infection from inital exposure to the onset of full blown AIDS (Acquired Immune Deficiency Syndrome) is known to occur over time $(t)$, with range $2 \leq t \leq 20$ years [1]. Computer models that successfully reproduce the behaviour of the HIV pathogen are extremely good at replicating the localized intra- and inter- cellular behaviour over a certain period of time and space. In particular, the affect of viral mutation on the immune response has been demonstrated by [2] and [10] while the work of [4] and [5] have shown, at least in outline, how bit-string models can represent the state of the immune repertoire. However, to our knowledge, there are no computer models that successfully address the issue of why it is that different immune systems respond differently to the same viral exposure. It follows from this that in addition to modelling intra-cellular localized interaction, there is a challenge to find a way to express the generalized distinction that exists between the immune systems of different people. In this paper we postulate that the course of an infection is crucially dependent on the density and distribution of the immune repertoire, and furthermore, that $\mathrm{T}$ cell receptor cross-reactivity is a crucial factor in determining the success or otherwise of the immune response to pathogen challenge. 


\section{Model}

The synthesis of two distinct approaches is the key to our model. Firstly, we model the physical space of the secondary immune organs (such as the lymph nodes or spleen) by way of two-dimensional stochastic cellular automaton [6] with periodic boundary conditions. This physical space model is the location in which host invading intra-cellular pathogens, having been engulfed by antigen presentation (APC) cells, are presented on the cell surface to naive recirculating cytotoxic T lymphocyte (CTL) cells. Upon recognition of a specific antigen, naive lymphocytes stop recirculating and undergo cell division. The process of cell division is limited to clones of the original lymphocyte that recognized the viral pathogen (this original lymphocyte is known as a CTL-precursor), and all cloned daughters inherit the specific $\mathrm{T}$ cell receptor that was successful in this recognition process. The daughters of the CTL-precursor cells will normally divide at a rate of 2-4 every 24 hours and this process continues for some 3-5 days [9]. This process is known as clonal expansion, and forms the basis of our physical space model. In our model, we assume that all antigen presentation cells carry the major histocompatibility complex (MHC) class I molecule which is required by CTL-precursors in order to produce armed effector CTL cells at the end of the clonal expansion process

The second component to our model is an implementation and extension of the shape space formalism originally presented by [7]. We utilize shape space to model the density and distribution of the T Cell Receptor (TCR) repertoire, and to lend analytical insight into the critical nature of the measure of crossreactivity, which we denote as $\rho$. In our model, shape space is a dynamic and evolving $N$-dimensional Euclidean space that contains one vector $\mathbf{u}$ for every immune system CTL-p genotype, and one vector $\mathbf{v}$ for every pathogen genotype. Around each $\mathbf{v}$ in shape space is a disc of influence of radius $\rho$. Any $\mathbf{u}$ falling inside this disc of influence is subject to some pressure. That is to say, the pathogen will be removed from the physical space system with some probability $P(X)$, inversely proportional to the distance between $\mathbf{v}$ and $\mathbf{u}$ in shape space. If the distance $d$ between the two points exceeds the critcal value $\rho$, then there is no CTL pressure on the pathogen, and no affinity or binding takes place. The process is summarized in equation (11) and equation (2)

$$
\begin{gathered}
d=\|\mathbf{v}-\mathbf{u}\|=\sqrt{\sum_{i=1}^{N}\left(v_{i}-u_{i}\right)^{2}} \\
P(X)=\left\{\begin{array}{lll}
1 & : & d=0 \\
\frac{1}{d} & : & 0<d \leq \rho \\
0 & : & d>\rho
\end{array}\right.
\end{gathered}
$$

It should be emphasized at this point that equation (11) and equation (2) will be evaluated if and only if there is some contact between an APC and a CTLp within the physical space model. Contact in this case means that within the physical space lattice the cells representing the CTL-p and the APC are adjacent 
- or are nearest neighbours. If equation (2) does result in the removal of the APC from the physical space, we use $P(X)$ as the stimulation rate $(S)$ for the exponential growth during the clonal expansion phase $(S \equiv P)$. Therefore, the total number of activated CTL daughter clones which the $i$ th CTL-p gives rise to, $\left(T_{C T L}^{i}\right)$ is given by:

$$
T_{C T L}^{i}=e^{0.036 \tau S}
$$

where the power of $e$ depends on three parameters, $\tau$, the duration of the expansion phase, $S$, the stimulation rate (dependent on the distance between the APC and CTL-p in shape space), and a constant factor 0.036. At the end of the clonal expansion phase, when $\tau \approx 192$ (representing some four days of actual time) and where $S=1$, we see some 1000 clones have been produced. This is what we would expect in a healthy immune system 9 . Clearly, the effect on $T_{C T L}^{i}$ when $S$ decline is noticeable, for example, for a case with weak stimulation, $S=0.3, T_{C T L}^{i} \approx 8$.

Our physical space model is implemented as a discrete two-dimensional array of C-language pointers to data structures. Each member of the array contains a pointer to it's own structure (even if the structure is logically empty). In turn, each structure contains a set of information which is summarized in Table 1 ] At each time step, we randomly select locations on the array to update. We ensure full coverage of the array by using a member of the structure to indicate that this array element has been updated. When $99 \%$ of the array has been visited, we reset the visited flag on each array member, and increment the clock counter by one, thus indicating we have moved on to time $\tau+1$. Our shape space model is implemented as a one-dimensional array (of length 2). As both APC and CTL-p cells have a representation in shape space, this value is again carried by each of the structures in the physical space array. In Table 1 the location of a given APC or CTL-p in shape space is referred to as sscoord. We implement motion on the lattice merely by swapping pointer references (thus, no expensive in-memory copy activities are required). For example, assume an infected APC cell is located at position $L_{i}$, and at position $L_{i+1}$ there is an unoccupied cell. We simply swap the pointers stored in $L_{i}$ and $L_{i+1}$ with each other, and the move is complete. For further treatment of physical space models see 3 . For other models of shape space, see [12].

\subsection{Model Parameters}

Although the model presented here is capable of supporting most of the known entities of the immune system, we restrict our inital study of the immune state to antigen presentation cells (APC), cytotoxic lymphocyte precursor (CTL-p) cells, and activated cytotoxic lymphocyte (CTL) cells. We justify the exclusion of other entities (such as Th cells, B cells and cytokines) by the fact that it is through activation and differentiation of CTL-p cells that the immune repertoire is known to evolve when the immune system is faced with an intra-cellular pathogenic challenge, such as the Human Immune Virus (HIV). Within our physical space model, we define $L_{p s}$ as the length of one side of the square lattice, $D_{c t l-p}$ as 
Table 1. Data structure maintained at each physical space array member

\begin{tabular}{lll}
\hline Data type & Parameter & Definition \\
\hline unsigned char act & Cell type \\
unsigned char state & Cell state \\
unsigned char sscoord[] & Location of cell genotype in shape space \\
unsigned int & ticks & The age of this cell \\
unsigned int & max-ticks & The maximum age of this cell \\
double & prolif-rate & The rate at which this cell proliferates $(0 . .1)$ \\
unsigned int & last-updated & Timestep when cell was last visited \\
unsigned int & updated-count cell visited flag \\
unsigned int & id & Unique ID for this cell \\
\hline \hline
\end{tabular}

the density of the CTL-p cells, $D_{a p c}$ as the density of the APC's. During each of the model simulation runs, we keep all parameters other than $\rho$ constant. The largest value of $\rho$ is known as $\rho_{\max }$, the derivation of which is shown in equation (4).

$$
\rho_{\max }=\sqrt{\frac{L_{p s}^{2}}{\pi}}
$$

We define $\rho_{\max }$ as a measure of the maximum cross-reactivity of a given genotype in shape space. Setting $\rho=\rho_{\max }$ would be equivalent to having every CTL-p cross-react with every APC. Although we do not explore this configuration further, it is worth noting that this configuration could represent a clinical condition known as auto-immune disease (wherein the immune system attacks both itself and invading pathogens without discretion). Auto-immune disease is a relatively rare condition $([13])$ and will be examined in further work. As we are initially interested in observing the behaviour of the model as $\rho \rightarrow \rho_{\max }$, we express the ratio of $\rho$ to $\rho_{\max }$ as $\hat{\rho}$, which takes values in the range $\mathcal{A}=\{0.5,0.29,0.10\}$. $\mathcal{A}$ represents one possible set of values for $\hat{\rho}$ in order of decreasing cross-reactivity. We could have chosen any set of values for $\hat{\rho}$ that follow $0 \leq \hat{\rho} \leq 1$. From equation (4), the value for $\rho_{\max }$ in our model is $\approx 28$. The model parameter initial values are summarized in Table 2 .

\section{Shape Space}

The shape space idea, as an underpinning of theoretical immunology, was introduced by [7] in 1979, but has attracted some criticism, notably from [16]. It is instructional at this point to review the basis of this important formalism and to address the comments of [16]. We will also justify our utilization of shape space while highlighting potential shortcomings.

In shape space, the antigenic determinant for a given antibody or antigen is an $N$-dimensional vector consisting of values representing such parameters as geometric configuration (at the molecular level), electric charge and other 
Table 2. Table of initial model parameters

\begin{tabular}{lll}
\hline Parameter & Definition & Initial Value \\
\hline$D_{c t l-p}$ & Density of CTL-precursors & 2000 \\
$\theta$ & Number of genotype CTL in shape space & 2000 \\
$D_{a p c}$ & Density of APC & 100 \\
$L_{p s}$ & Length of one side of physical space square lattice & 100 \\
$L_{s s}$ & Length of one side of shape space square lattice & 50 \\
$\tau$ & Number of time steps per simulation run & 300 \\
$\rho_{\text {max }}$ & Max. cross-reactivity of shape space genotype & $\approx 28$ \\
$\mathcal{A}$ & Range of $\hat{\rho}$ & $\{0.5,0.29,0.1\}$ \\
\hline
\end{tabular}

complex chemical characteristics that are not postulated in [7]. According to this approach, not all elements of the $N$-dimensional vector play an equal role in determining complementarity or match. Given an antibody vector $\mathbf{A b}$ and an antigen vector $\mathbf{A g}$, if $\mathbf{A b}=\mathbf{A g}$ then the two entities will bind absolutely. If we now assume that the $N$-dimensional vector represents a location in an $N$-dimensional Euclidean space, then

$$
\|\mathbf{A b}-\mathbf{A g}\|=0
$$

means that $\mathbf{A b}$ and $\mathbf{A g}$ are coincident in the space (that is, they share the exact same coordinates within the space), and this space is known as shape space. Therefore, we can say that shape space is the space of all possible vectors each one representing a unique set of antigenic determinants (or shapes). One of the criticisms that [16] level at the above theory is that the function $f(\mathbf{A b}, \mathbf{A g})$, which, according to [7, will determine the 'distance' (and hence, affinity) between $\mathbf{A b}$ and $\mathbf{A g}$, must be highly irregular and discontinuous. This assertion is based on work by chemists which has shown that predicting affinity and bonding between two molecules is not simply a deterministic issue of understanding the dynamics between the individual molecular constituents. However, [7] clearly comment towards the end of their paper that shape space does not need to be uniform (that is, there does not have to be a uniform distribution of $\mathbf{A b}$ vectors within Euclidean space). Therefore, $f$ does not need to be either continuous or regular. In fact, 7] offer a model for representing the probability of detecting antigens in a non-uniformly distributed shape space.

Further criticism of the shape space paradigm is the question of the value for $N$. Although [16] insist the original value of $5 \leq N \leq 10$ is too small (they suggest a value closer to $N=20$ ), they do not, in principle, question the theoretical foundation of representing antigenic determinants by a fixed, $N$-sized set of parameters. The actual value for $N$ is clearly something that is system specific and may vary. In fact, [17] have shown how the presence of cytokine regulatory molecules crucially affects the dynamics of helper $\mathrm{T}$ cell populations. It is therefore plausible that the actual value and parameters of $N$ are not only dependent on the characteristics of $\mathbf{A b}$ and $\mathbf{A g}$, but also of external and local- 
ized state information such as the density of cytokines. It seems reasonable to conclude that setting $N$ to a fixed and relatively small number is sufficient to represent the parameters of antigenic determination.

Finally, to conclude this section, we address the specific utilization of the shape space paradigm within the model presented in this paper. Firstly, we do not require or assume a continuous $\mathbf{A b}$ distribution across our shape space. The probability that an APC will fall into a region for which equation (1) is not defined (and hence, not be under any CTL pressure) is given by

$$
P(\bar{X})=1-\frac{\sum_{i=1}^{\theta} \pi \rho^{2}}{L_{s s}^{2}}
$$

In our model we assume that shape space is populated by a uniform distribution of genotype CTL cells. It can be seen from equation (6) that $\rho$ is a critical parameter. Additionally, in a healthy immune system, equation (6) would always result in a value close to zero. Secondly, we extend the original shape space formalism by introducing a stimulation rate which is inversely proportional to the distance as calculated using equation (1). This partially addresses the deficiencies in other shape space -based models, most notably that of 12 . Thirdly, with respect to the value for $N$, although we set $N=2$, this is by no means a hard parameter of our model. As $N$ represents the dimensionality of the shape space, we should, in principal, set $N$ to a value such that $L_{s s}^{N} \rightarrow \infty$ (as the number of antigenic determinants that might be presented to the immune system is, essentially, infinite). For practical computational purposes, such a space would require resources beyond current computing boundaries available today. As the research presented here studies the affect of specific pathogen detection and removal, our space needs only to accomodate antigenic variation for a specific strain, and thus $N=2, L_{s s}^{N}=2.5 \times 10^{3}$ is sufficient for our initial purposes.

\section{Results}

Fig. 1 shows the state of three immune TCR shape spaces at time $\tau=300$ (where $\tau=1$ models 30 minutes of elapsed time), and Fig. 2] shows the denstity of APC in the lymph system over time $0 \leq \tau \leq 300$ for each of the three immune system configurations. The only parameter altered across each of the three configurations is the ratio of $\hat{\rho}$, the values of which are drawn from $\mathcal{A}$ (and are represented in the figures by the diamond, the circle and the square, respectively). We notice, in Fig. 11 that the shape space has evolved into three quite different states. The first state can be classified as healthy in the sense that the clearance rate of APC from the lymph system is broadly in keeping with what is known to be the case clinically [9] (and represented here as the diamond in Fig. 2). We can see that the healthy shape space state can be characterized as a low affinity, low density configuration. This is in agreement with [8]. As the immune cross-reactivity declines (represented by the circle, Fig. 11 and 2]) we see an apparently paradoxical condition in the shape space realization. That is, as 
promiscuity diminishes, it takes greater specificity (and hence, greater stimulation) in order to clear APC. In fact, we can see from Fig. 2 that a $90 \%$ clearance from the lymph system takes some $\tau=300$ time steps. We conclude that the immune system configuration, with reduced cross-reactivity, is chronically unable to clear all APC and is operating at a sub-optimal level. Finally, when we test the immune configuration at the lowest level of cross-reactivity (represented by the square) - we see a very strong and highly specific four receptor response in shape space. Having reduced promiscuity to its lowest level, the effect on APC clearance is conspicuous. From Fig. 2 we can see only a $10 \%$ APC removal from the lymph system after some 6 days. It is clear that were this the immune system of a real person, it would be at the point of collapse, and hence could represent the onset of full-blown AIDS (wherein the patient dies by way of opportunistic infection).

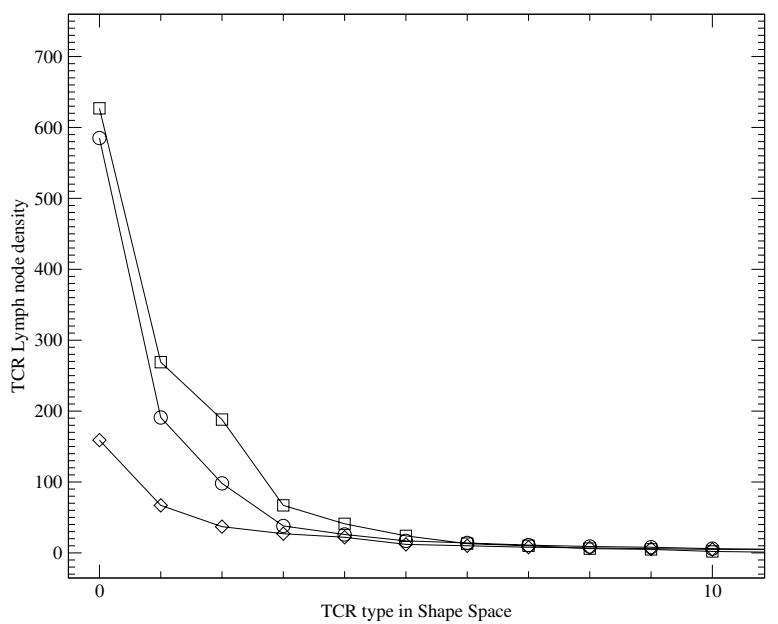

Fig. 1. Immune repertoire density and distribution for 3 values of $\hat{\rho}$ drawn from $\mathcal{A}$, with $\tau=192$. Shown here are the density levels for the ten most dominant TCR types in shape space. The healthy state of the system (diamond) is a low-affinity, low average density configuration. As affinity increases and promiscuity declines, a more specific and active repertoire evolves. The state represented by the square is the least healthy, and represents an immune system at the point of failure.

\section{Discussion}

Our starting point for the experiments presented here is to assume that the immune repertoire has not been exposed to any prior pathogen. Hence, we do not model immunological memory or reinfection. Our objective has been to study how insight (into different IS evolutionary states) can be gained by integrating localized and generalized models of immune response. Informally, our theoretical 


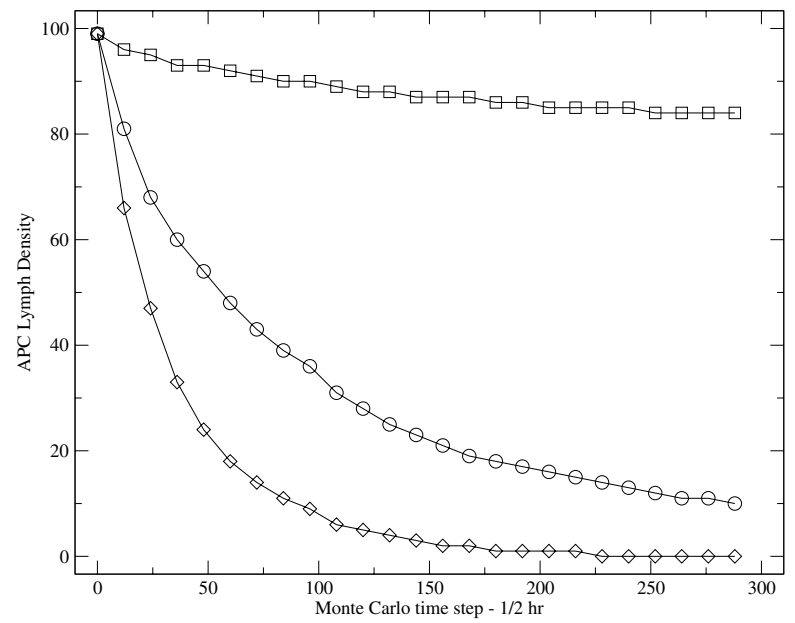

Fig. 2. Clearance rate of APC's from the lymph node system over about 6 days $(\tau=$ 300) for various values of $\hat{\rho}$ drawn from $\mathcal{A}$. The three distinct states which have resulted: healthy, chronic and fatal (diamond, circle and square, respectively). These states show the affect of decreasing $\hat{\rho}$ on APC clearance.

expectation is that by studying the evolution of shape space, we can demonstrate how specific configurations of the repertoire occur. Following from this, we can also show one possible mechanism for the development of immunodominance 1. With reference to Fig. 1, we see that immunodominance becomes more pronounced as cross-reactivity declines. An immune system with high levels of immunodominance gives rise to an inefficient detection and clearance regime. Therefore, when we examine the difference in infection outcome (Fig. 2] we notice that a more strain-specific response results in poorer short-term disease detection and clearance. Although we have not modelled pathogenic mutation in this model, we can hypothesize that the repertoire configurations that have evolved might play a role in determining the dynamics of a virus mutation and immune clearance. We feel the model presented here is a sound basis for exploring such issues in the future.

\section{Conclusion}

A model of the immune system is presented which utilizes a new extension to the shape space formalism, and addresses - at least in part - some of the former criticisms. We argue for the value of a shape space formalism for modelling some aspects of the immune repertoire. We have presented initial results from the model that suggest that a low-affinity $\mathrm{T}$ cell receptor (TCR) space provides the

\footnotetext{
${ }^{1}$ Immunodominance is the process by which a small number of specific TCR's are reponsible for clearing an antigen or virus
} 
most efficient APC removal. Our findings are in agreement with recent work presented by [8]. We have shown that, for all other model parameters being unchanged, reducing TCR promiscuity causes significantly different evolution of the shape space and correspondingly poorer APC removal. We hypothesize that $\rho$ (the cross-reactivity measure) is one of the most crucial general parameters within the immune system. We have demonstrated, for one configuration of the model, results which are similar in signature to those which characterize the onset of AIDS. A major factor underlying the evolution of an individual's immune system (and its overall health) appears to be the role played by crossreactivity, $\rho$. For $\hat{\rho}<\hat{\rho}_{\text {crit }}$ (where $\hat{\rho}_{\text {crit }}$ is crudely estimated to be in the range $\left.0.29 \leq \hat{\rho}_{\text {crit }} \leq 0.1\right)$, the immune system is essentially undermined and faces almost immediate collapse. For more viable values of $\hat{\rho}\left(0.5<\hat{\rho}_{\text {crit }}<0.29\right)$ the residual antigen cells are maintained at a limited levels in every case, with less than $100 \%$ effective clearance.

\section{References}

1. Nowak M., McMichael A. J.: How HIV defeats the Immune System. Scientific American 273 (1995) 58-65

2. Mannion, R., Ruskin, H., Pandey, R.B.: Effect of Mutation on Helper T-cells and Viral Population: A Computer Simulation Model for HIV Theor. in Biosci. 119/2 (2000) 145-155

3. Seiden, P., Celada, F.: A model for simulating cognate recognition and response in the immune system, J.Theor. Biol. 158 (1992) 329-357

4. Lagreca, M.C., de Almeida, Zorzenon dos Santos, R.M.C.: A dynamical model for the immune repertoire. Physica A 289 (2001) 191-207

5. Castiglione, F., Motta, S., Nicosia G.: Pattern recognition by primary and secondary response of an Artificial Immune System. Theory Biosci. 120 (2001) 93-106

6. Wolfram, S.: Cellular Automata as Simple Self-Organizing Systems. Nature (1982)

7. Perelson, A. Oster, G.: Theoretical Studies of Clonal Selection: Minimal Antibody Repertoire Size and Reliability of Self-Non-self Discrimination, J. Theor. Biol. 81 (1979) 645-667

8. van den Berg, H.A., Rand D.A., Burroughs, N.J.: A Reliable and Safe T Cell Repertoire based on Low-affinity T Cell Receptors. J. Theor. Biol 209 (2001) 465486

9. Janeway, C.A., Travers, P., Walport, M., Capra, J.D.: Immunobiology. The Immune System in Health and Disease. Churchill-Livingston 4th Edition (1999)

10. Zorzenon dos Santos, R.M., Coutinho, S.C.: Dynamics of HIV Infection: A Cellular Automata Approach. Phys. Rev. Lett. 87 (2001) 168102

11. Nowak, M., May, R.: Mathematical Biology of HIV Infections: Antigenic Variation and Diversity Threshold. Mathematical Biosci. 106 (1991) 1-21

12. Hershberg, U., Louzoun, Y., Atlan, H., Solomon, S.: HIV time: winning the war while, loosing all the battles. Physica A 289 (2001) 178-190

13. Orosz, C.: An Introduction to Immuno-ecology and Immuno-informatics. Design Principles for Immune System and Other Distributed Autonomous Systems. Oxford University Press (2001)

14. Monteiro, L.H.A., Goncalves, C.H.O., Piqueira, J.R.C.: A condition for Successful Escape of a Mutant after Primary HIV Infection. J. Theor. Biol. 203 (2000) 399406 
15. Nowak, M., May, R.: Virus Dynamics. Mathematical Principles of Immunology and Virology. Oxford University Press (2000)

16. Carneiro, J. and Stewart, J.J.: Rethinking "Shape Space": Evidence from Simulated Docking Suggests that Steric Complementarity is not limiting for AntibodyAntigen Recognition and Idiotypic Interactions. J. Theor. Biol. 169 (1994) 391-402

17. Yates, A, Bergmann, C., Van Hemmen, J.L., Stark, J., Callard, R.: Cytokinemodulated Regualtion of Helper T Cell Populations J. Theor. Biol. 206 (2000) $539-560$ 